Revista Complutense de Historia de América ISSN: 1132-8312

http://dx.doi.org/10.5209/RCHA.61079

\title{
Etnografía en un fondo histórico. La mano de obra y las inquietudes de la Casa Suárez ante la crisis gomera, 1920-1940
}

\author{
Kathryn Lehman ${ }^{2}$
}

Recibido: 13 de noviembre de 2017 / Aceptado: 3 de junio de 2018

Resumen. Este artículo examina la Casa Suárez durante el período de crisis de la economía de la goma elástica, después del año 1920. Tomando como herramienta de análisis el método de «etnografía en y del archivo» propuesto por Laura Ann Stoler (2009), se estudia el modo en que las inquietudes relativas a la inseguridad territorial y las dificultades económicas sufridas entre 1920 y 1940 afectaron la percepción de la empresa gomera hacia sus trabajadores. En estos tiempos de incertidumbre, la administración de Casa Suárez percibió al conjunto de sus trabajadores gomeros como herramientas para combatir los problemas que la administración enfrentaba. Como consecuencia, la administración realizó cambios en las representaciones de la fuerza laboral, que se encuentran plasmadas en los documentos internos de la Casa, dejando de ser vistos los trabajadores como elementos que debían producir la mayor cantidad de goma posible para pasar a ser considerados como un colectivo al que había que mantener y proteger. Palabras clave: Representaciones; etnografía en el archivo histórico; adaptación; trabajadores gomeros; Amazonía; siglo XX.

\section{[en] Ethnography in the Historical Archive. Workers and Anxieties of the Casa Suárez in the Face of the Rubber Crisis, 1920-1940}

\begin{abstract}
This article examines the Casa Suárez during the crisis of the rubber economy. Using Laura Ann Stoler's (2009) method of «ethnography in and of the archive» as an analytical tool, I study the way in which the anxieties suffered between 1920 and 1940 regarding territorial insecurity and economic difficulties, affected the Casa's perceptions of its workers. During these times of uncertainty, the Casa Suárez administration perceived all its workers as tools for combating the problems they faced. As a consequence, there was a change in the representations of the labour force, expressed, in the documents of the Casa Suárez. Workers were no longer seen as elements that had only to produce as much rubber as possible, rather as an occupying population that had to be maintained and protected.
\end{abstract}

Keywords: Representations; Ethnography in the Archive; Adaptation; Rubber Workers; Amazonia; 20th Century.

Sumario. 1. Introducción. 2. La situación geopolítica de la Casa Suárez y la pérdida del Acre. 3. Trabajadores de la Casa Suárez y el régimen de trabajo. 4. Crisis y adaptación. 5. Conclusiones. 6. Referencias bibliográficas.

1 Agradezco a la Dra. Anna Guiteras Mombiola por sus útiles sugerencias y revisiones y también a los tres evaluadores anónimos de la Revista Complutense de Historia de América. El presente trabajo fue elaborado con el apoyo de la oficina del Provost para asuntos internacionales de Indiana University (Bloomington, USA), la Fundación Tinker y la Fundación Mellon.

2 Indiana University, Bloomington, Indiana (Estados Unidos)

E-mail: kaelehma@indiana.edu 
Cómo citar: Lehman, K. (2018) Etnografía en un archivo histórico. La mano de obra y las inquietudes de la Casa Suárez ante la crisis gomera, 1920-1940, en Revista Complutense de Historia de América $\mathrm{n}^{\mathrm{o}} 44,47-66$.

\section{Introducción}

Durante la segunda mitad del siglo XIX y primer tercio del siglo XX, centenares de trabajadores procedentes de distintas partes de Bolivia y Brasil se desplazaron a la región del Alto Madera para incorporarse como mano de obra en las concesiones de goma elástica que ambos Estados habían otorgado a una multiplicidad de empresarios. En Brasil, escapando de las sequías que afectaban la región de Sertão, los nordestinos fueron extendiéndose a lo largo y ancho de la cuenca amazónica en busca de trabajo en las numerosas barracas gomeras que se habían establecido en la región desde $1850^{3}$. En Bolivia, en los primeros años de la economía gomera, el mayor contingente de mano de obra procedió de los antiguos centros misionales situados en los llanos de Mojos ${ }^{4}$. Posteriormente, cuando sobrevino el auge gomero, muchos trabajadores llegaron desde las varias regiones del Oriente boliviano. Tanto indígenas como mestizos del norte paceño, las llanuras cruceñas, la Cordillera chiriguana e, incluso, la misma ciudad de Santa Cruz de la Sierra fueron reclutados como mano de obra, voluntaria o forzada, y enviados a trabajar la goma en los bosques del noroeste ${ }^{5}$.

Para entonces, la Casa Suárez, empresa gomera dirigida por Nicolás Suárez, había establecido el monopolio sobre la producción y comercialización de la goma elástica del noroeste amazónico boliviano ${ }^{6}$. La empresa poseía más de la mitad de las tierras que el gobierno había adjudicado en toda la región, tanto en propiedad definitiva como en simple concesión, y empleaba una gran parte de la población regional. Paralelamente, la presencia del Estado boliviano en el área amazónica era muy marginal. Ello respondía al hecho que, históricamente, los grupos dirigentes habían orientado la economía nacional hacia la extracción de la plata y el estaño, interesándose en la población concentrada en los centros de minería altiplánicos ${ }^{7}$. Además, la región gomera boliviana estaba muy alejada de los centros de poder y su difícil acceso desde los mismos encarecía el transporte hasta la región. En este sentido, se podría decir que la Casa Suárez funcionó como un "para-Estado".

Al finalizar el siglo XIX, la jurisdicción boliviana sobre el norte amazónico se extendía hasta el curso de los ríos Acre y Purús. Sin embargo, la baja densidad de población de origen boliviano y la falta de una soberanía estatal efectiva en la región favorecieron que siringueros ${ }^{8}$ brasileros ocuparan buena parte de estos territorios, lo que daría lugar a la conocida como guerra del Acre (1899-1903). El conflicto por el

3 Sobre la historia de la goma y los siringueros brasileños nordestinos ver Costa Sobrinho, 1992; Martinello, 2004; Tocatins, 1961.

4 Guiteras Mombiola, 2012: 71-92; Van Valen, 2013: 65-99.

5 Roca, 2001: 109-119; Vallvé, 2010: 288-377; Córdoba, 2012.

6 En la Amazonía existen distintas variedades de árboles de goma elástica. La especie Hevea Brasilensis es la más valiosa y se encuentra en grandes concentraciones en la región del Alto Madera y Alto Purús, área del presente estudio.

$7 \quad$ Fifer, 1972; García Jordán, 2001: 360-366.

8 La palabra "siringuero" (seringueiro en portugués) se refiere a aquella persona que trabaja en la recolección de Hevea Brasilensis -siringa en Brasi1* de los árboles gomeros, conocidos también como siringueras. 
Acre con Brasil y otras escaramuzas con contingentes peruanos durante la misma época en el alto Madre de Dios ${ }^{9}$ tuvieron un impacto duradero en la Casa Suárez y su propietario, don Nicolás Suárez. Finalizado el enfrentamiento armado, la incapacidad del Estado boliviano por controlar efectivamente el noroeste y la presión brasileña sobre los límites fronterizos persistieron. Esta situación contribuiría al surgimiento de cierto clima de inseguridad entre los bolivianos de la frontera, particularmente entre los administradores de la Casa Suárez; incertidumbre que se intensificaría tras la fuerte caída del precio de la goma en el mercado internacional en el año 1912 que causó una profunda crisis social y económica en la Amazonía boliviana, después del año de 1920.

En este contexto, los trabajadores de la Casa Suárez fueron concebidos como algo más que una fuente de producción económica: su fuerza laboral devino el principal recurso para poblar el norte amazónico y, así, proteger el país del expansionismo brasilero. La gran mayoría de estos trabajadores eran analfabetos y, en consecuencia, dejaron muy poco registro de sus experiencias ${ }^{10}$. Pese a ello, es posible rastrear sus vidas en los expedientes de la empresa. Esta documentación se encuentra actualmente en el Archivo Histórico Casa Suárez, en Guayaramerín (Beni) ${ }^{11}$. En un espacio donde se hallan disponibles pocas otras fuentes escritas, este archivo proporciona un importante registro del funcionamiento de este mundo laboral y del modo en que fueron percibidos y representados sus trabajadores.

Toda representación hecha por otra persona está condicionada $-\mathrm{y}$ es indicativa de una diversidad de factores que convergieron durante su producción. Así, como señala Roger Chartier, el concepto de la representación permite aplicar la historia cultural para comprender cuestiones sociales como las relaciones de poder y las relaciones laborales. Un análisis a través de los procesos de representación permite abordar la producción de clasificaciones y exclusiones, así como las relaciones de poder existentes en el momento de la producción de un texto ${ }^{12}$. Éstas últimas son esenciales para comprender tanto el desarrollo de los acontecimientos en el pasado, como el modo en que fueron registrados. La producción documental -base de nuestra comprensión del pasado- y de narrativas históricas implica, según palabras del historiador y antropólogo Michel-Rolph Trouillot ${ }^{13}$, una contribución irregular por parte de grupos e individuos en competencia y con acceso desigual a los medios para dicha producción.

Como han señalado Vallvé y Córdoba ${ }^{14}$, la gran mayoría de los trabajadores de la Casa Suárez no tenían acceso a los medios de producción de sus propias representaciones. De ahí que, por lo general, todo acercamiento a grupos subalternos como

9 Una visión concisa del conflicto con Perú en el norte de Bolivia, en Fifer, 1972: 141-150.

10 Sí existe registro de otra clase de trabajadores -empleados, administradores y gerentes- en textos escritos por ellos mismos. Ver Córdoba, 2015.

11 Entre junio y julio de 2013 estuve trabajando en el Archivo Histórico Casa Suárez (en adelante ACS) [Bolivia]. La inexistencia de un catálogo de su contenido dificultó la realización de un trabajo sistemático y exhaustivo con las fuentes que custodia. Varias cajas y paquetes carecían de referencias numéricas y/o descriptores y, en algunos casos que sí las tenían, éstas no correspondían con los documentos preservados en su interior. No existía tampoco un orden lógico -cronológico, temático- de la documentación en el almacenamiento en los estantes del archivo. Asimismo, las fuentes presentaban un estado físico bastante precario al estar llenas de polvo y dañadas por insectos y la humedad, debido a la falta de climatización -por entonces la energía eléctrica del edificio se encontraba cortada y las ventanas permanecían abiertas.

12 Chartier, 1988: 13.

13 Trouillot, 1997: xix.

14 Vallvé, 2012; Córdoba, 2012. 
éstos se haga siguiendo sus rastros de agencia y resistencia. Así, inspirado por el estudio clásico Weapons of the Weak de James Scott (1985), Frederic Vallvé muestra que "las formas de resistencia desarrolladas por los siringueros [...], aunque circunscritas, pueden interpretarse como intentos de afianzar su representación dentro de la industria gomera y optimizar sus condiciones de trabajo"15.

Por el contrario, aquí interesa el cambio que se produjo en la concepción de la mano de obra de la Casa Suárez como consecuencia de los reajustes laborales que ésta se vio obligada a introducir a partir de las décadas de 1920 y 1930. La lectura atenta de la correspondencia y los expedientes internos de la empresa revela lo que Ann Laura Stoler ha denominado "el espacio psíquico del imperio"16. Por medio del uso de la etnografía de y en el archivo como metodología de análisis, Stoler analiza lo que ella termina como inquietudes [anxieties] epistémicas y políticas cuando analiza el caso del imperio colonial holandés plasmadas en la documentación producida durante su declive. Tomando parte de esta metodología para el estudio del imperio gomero de la Casa Suárez en Bolivia, se muestra que los cambios en la imagen que se tenía de sus trabajadores fueron ocasionados y, a la vez, son ilustrativos de las inquietudes y temores de la empresa en relación a la soberanía nacional y la seguridad territorial. En otras palabras, propongo un análisis de la construcción y modificación de la representación de la mano de obra en un contexto de incertidumbre. Los documentos contenidos en el Archivo Histórico Casa Suárez (correspondencia, escritos, memorándums) son escritos por los empleados de mayor categoría, como los administradores de los establecimientos, los administradores de la casa matriz en Cachuela Esperanza, los contadores y los mayordomos, entre otros. Ellos son, frecuentemente, los autores de la documentación de la empresa y, por consiguiente, son quienes escriben sobre los trabajadores de la Casa que estaban a su cargo. En suma, sus palabras nos revelan las actitudes y las percepciones que tenía la empresa en lo que se refiere a su fuerza laboral.

A través del análisis de la documentación interna de la Casa Suárez elaborada entre las décadas de 1920 y 1940, en un primer apartado se describirá la situación geopolítica histórica del norte de Bolivia a inicios del siglo XX y en un segundo apartado se identificarán las principales características de los trabajadores tales como los mozos, siringueros y fregueses. En un tercer apartado, se analizará el impacto de la caída del precio de la goma en la Casa Suárez y las adecuaciones que adoptó - en lo económico y en lo laboral- para sobrevivir a esta crisis que evidenciaron un cambio de percepción respecto sus trabajadores, pasando de una perspectiva básicamente orientada hacia el beneficio económico a una que apostaba por el mantenimiento de una población de trabajadores como un fin en sí mismo.

\section{La situación geopolítica de la Casa Suárez y la pérdida del Acre}

El boom de la goma en la Amazonía fue el primer proceso histórico que estableció vínculos significativos y duraderos entre los remotos sistemas fluviales del Madera, el Purús y el Acre con la economía mundial a través del río Amazonas. Atrajo, también, un número significativo de colonizadores bolivianos, brasileños y peruanos, y

Vallvé, 2012: 64.

Stoler, 2009: 25. 
captó la atención de los gobiernos de los tres países por primera vez ${ }^{17}$. Hasta el último tercio del siglo XIX, el Acre persistió como "tierra de nadie" ya que las casas comerciales brasileñas tenían sus sedes en Manaos y Pará, a orillas del río Amazonas, mientras que las operaciones de goma bolivianas se centraban alrededor de Riberalta y Cachuela Esperanza y en los ríos Beni, Madre de Dios y Orthon.

De acuerdo con los tratados existentes entre Bolivia y Brasil, las cabeceras de los ríos Acre y Purús y la cuenca del Madera recaían sobre el lado boliviano de la línea que separaba ambos países ${ }^{18}$. A pesar de ello, en 1880 los ríos Acre y Purús estaban poblados mayoritariamente por indígenas independientes sin ninguna afiliación a los dos países y por siringueros brasileños que buscaban explotar siringa en los bosques vírgenes ${ }^{19}$. Las empresas bolivianas trataron de enfrentar la enérgica penetración de los intereses brasileños con escasos resultados ${ }^{20}$. La escasez de mano de obra en la región se tornó crónica debido a la difícil accesibilidad de los bosques gomeros, lo que requería de enormes esfuerzos (humanos y económicos) para atraerla desde otras partes ${ }^{21}$. Por el contrario, las sequías que afectaron el nordeste brasileño en la segunda mitad del siglo XIX fomentaron el desembarco de un gran número de sus habitantes en la Amazonía ${ }^{22}$. Sin obstáculos significativos como los rápidos de la vía Madera/Mamoré o los largos acarreos terrestres que debían superar los bolivianos, los nordestinos llegaron sin dificultad al Acre por la vía del Amazonas y luego por los ríos Purús y Acre. La goma extraída en territorio boliviano empezó a fluir libremente hacia Brasil sin pagar impuestos de exportación a Bolivia. Además de las pérdidas económicas que ello suponía, la presencia de brasileños en la frontera representaba una amenaza geopolítica que cuestionaba la soberanía boliviana en la región. En vano se crearon en la década de 1890 las delegaciones del Acre y Purús y del Madre de Dios, unidades administrativas que, junto con la aduana de Puerto Alonso, debían evitar el contrabando en la zona. Tras la fallida declaración de independencia del Acre en $1899^{23}$, el recién establecido gobierno del partido liberal de José Manuel Pando invocó tres métodos para intentar consolidar el control de Bolivia sobre el territorio acreano: la creación del Territorio Nacional de Colonias en el Noroeste sobre la jurisdicción de las antiguas delegaciones nacionales, la ocupación y explotación del Acre por medio de un contrato con "The Bolivian Syndicate" de Estados Unidos y la creación del Vicariato del Acre ${ }^{24}$. Acciones que tuvieron por resultado una nueva rebelión en el Acre y un renovado estímulo en la región por parte del gobierno brasileño.

En este contexto, la Casa Suárez surgió como la fuerza dominante del comercio de la goma en Bolivia. Fundada y dirigida por Nicolás Suárez Callaú (1851-1940),

Fifer, 1970: 126-128; García Jordán, 2001: 380-384.

18 El tratado de Ayacucho (1867) ratificó la soberanía boliviana sobre el territorio del Acre ya establecida por los antiguos tratados de Madrid (1750) y San Ildefonso (1777) que habían establecido una línea muy indefinida sobre las inexploradas regiones del Acre y Purús para separar los imperios español y portugués. La falta de definición sería la causa de los conflictos entre Bolivia y Brasil de inicios del siglo XX.

19 Tocantins, 1961.

20 Fifer, 1970: 127.

21 Pacheco, 1992: 70-75.

22 Cunha, 1976; Tocantins, 1978.

23 En 1899 un grupo de siringueros brasileños liderados por Luis Gálvez, expulsaron las autoridades de la aduana de Bolivia en Puerto Alonso y declararon la independencia del Estado del Acre. Al poco, las fuerzas brasileñas restablecieron el orden y su líder, quien ejerció brevemente de emperador del Acre, fue enviado a Fortaleza.

24 Fifer, 1972; García Jordán, 2001: 382-383. 
se trató de un negocio familiar integrado verticalmente en el que sus hermanos supervisaban diversos aspectos de la explotación y comercialización de la corteza de quina $^{25}$, primero, y la goma, después. La Casa Suárez articuló un enorme aparato que involucraba finanzas internacionales, transporte, bienes de consumo y servicios varios. Inicialmente centrada en el suministro de mercancías y transporte a otros empresarios $^{26}$, la empresa rápidamente estableció sucursales en Brasil -Santo Antonio, Manaus y Belem- y Europa -Londres-. Ello le permitió controlar el recorrido completo de su producción desde la sede central Cachuela Esperanza, en el departamento del Beni, y el río Amazonas hasta el Atlántico; y, a su vez, supervisar la compra y el transporte por la misma vía de las mercancías importadas. Paralelamente, la Casa diversificó sus intereses económicos: cría de ganado en Trinidad (capital del Beni) provisión de víveres y eventuales trabajadores a los gomales y servicio de transporte e importación hacia el interior. Estas actividades favorecieron la acumulación de capital que los Suárez solían invertir en más bienes e infraestructuras para la producción de goma ${ }^{27}$. Coincidimos, entonces, con Valerie Fifer ${ }^{28}$ cuando afirma que la palabra "imperio" es el término más apropiado para describir este negocio familiar.

El imperio de la Casa Suárez se encontraba geográficamente dispersado por todo el norte amazónico. Estaba compuesto por establecimientos o barracas y centros gomeros cuya ubicación venía determinada por la disposición de los árboles de goma en medio del bosque (las llamadas "estradas gomeras") y los ríos, el principal medio de transporte de la región ${ }^{29}$. Los asentamientos más grandes estaban situados a orillas de los ríos más importantes: Madre de Dios, Beni, Mamoré, Tahuamanu, Orton, Abuná, Acre, Manuripi. De ellos partían senderos o corrientes fluviales más pequeñas que los unían a los centros gomeros que, a su vez, se conectaban con las estradas, dando lugar a la ocupación dispersa de grandes extensiones territoriales que caracterizó a la Casa. Los asentamientos variaban mucho en tamaño, y los más grandes servían como puntos administrativos de nivel medio. Todos ellos contaron con personal administrativo que informaba a la dirección, en la sede central de Cachuela Esperanza ${ }^{30}$. Las barracas de mayor importancia tenían sus propias granjas para producir alimentos y fungían como centros de transporte, puntos de distribución de mercancías y puntos de almacenamiento de goma ${ }^{31}$. Entre estos establecimientos se destacaban El Sena y El Carmen, ambos ubicados en el río Madre de Dios, Humaitá, en el Rio Orton, y Filadelfia y Porvenir, en el río Tahuamanu. Este último río tuvo un significado especial para la Casa Suárez al tratarse del último río en el sentido noroeste que vierte sus aguas en el río Orton para luego juntarse sucesivamente a los ríos Madre de Dios y Beni, desde donde se incorpora al río Madera y, de ahí, al entramado del río Amazonas. Gracias a estas conexiones y en ausencia de vinculación caminera, las barracas de Filadelfia y Porvenir, tuvieron una ruta de acceso directo a la casa matriz, en Cachuela Esperanza. En contraste, el río Acre vierte sus aguas ha-

25 La corteza de quina, también llamada chinchona, se extraía de un árbol que se encuentra en las regiones amazónicas de los países andinos, entre ellos Bolivia. La corteza de este árbol contiene quinina y se usa para tratar la malaria. Véase Roca, 2001: 201-205; Vallvé, 2010: 115-128.

26 Gamarra, 2007: 354-395.

27 Pacheco, 1992: 95-98; Stoian, 2005: 64; Gamarra, 2007: 354-395.

28 Fifer, 1970: 130.

29 Gamarra, 2007: 227-228.

30 Córdoba, 2015; Gamarra, 2007.

31 Los organigramas del modelo administrativo clásico desarrollado por la Casa Suárez -conocido como "modelo empresarial a gran escala” en Gamarra, 2007: 227-228. Véase también Pacheco, 1992: 85-86. 
cia el norte, al complejo hidrográfico del Purús que desemboca en el Amazonas por otro punto. Esto hacía que el territorio del Acre y, más concretamente, las barracas de la Casa Suárez ahí ubicadas estuvieran desligadas del resto del espacio gomero boliviano y sus principales centros de poder. Sirva de ejemplo el hecho que Porvenir, situada en el río Tahuamanu, se conectaba únicamente por una senda de carretón de unos $35 \mathrm{~km}$. con la barraca de Bahía ${ }^{32}$, también propiedad de los Suárez y situada en la orilla sur del Rio Acre ${ }^{33}$.

Las características geográficas, hidrográficas y poblacionales del territorio del Acre jugarían un papel primordial en el desenlace del conflicto que enfrentó Bolivia y Brasil por el control de este espacio. La Guerra del Acre duró desde la rebelión de 1899 hasta la anexión del Acre por Brasil en $1903^{34}$. Tras la vida efímera del Estado independiente del Acre, surgió un nuevo personaje, Plácido de Castro, quien llegó a controlar el territorio acreano hasta las cabeceras del río Acre. Alegando que, por un lado, el contrato del Estado boliviano con "The Bolivian Syndicate" posibilitaba la invasión norteamericana del Acre y, por otro lado, que la mayoría de personas que vivían y trabajaban en dicho territorio eran brasileños, lideró una fuerza de siringueros que se enfrentó a las tropas bolivianas y al personal de la Casa Suárez ${ }^{35}$. El rol de Nicolás Suarez durante el conflicto fue muy significativo dado que la defensa de la soberanía boliviana en la región recayó, en un primer momento, en sus manos. La Casa Suárez movilizó un gran número de sus trabajadores para enfrentar las arremetidas brasileñas, además de entregar municiones, prestar sus establecimientos y ofrecer servicios de transporte al ejército boliviano. Pero, sin duda, su acción más reconocida fue la organización, con su propio personal, de la Columna Porvenir, surgida en la barraca del mismo nombre. Suárez adquirió gran renombre a nivel nacional al liderar, a la cabeza de esta columna, la defensa de la barraca Bahía, ocupada por tropas brasileñas. La victoria que lograría la Columna Porvenir en la llamada "batalla de Bahía" permitió que Bolivia mantuviera jurisdicción sobre el área que se extiende entre los ríos Acre y Madre de Dios. A su vez, el acontecimiento se convirtió en un elemento definitorio de la historia e identidad locales ${ }^{36}$. Tras el conflicto, Nicolás Suárez afirmaría que sus contribuciones en el conflicto del Acre daban cuenta de su patriotismo y se erigía en máximo defensor de la bolivianidad en la Amazonía ${ }^{37}$.

La guerra del Acre concluyó con la cesión, por parte de Bolivia, de 120.000 km² de territorio que actualmente constituye el estado brasilero del Acre ${ }^{38}$ a cambio, por parte de Brasil, de una compensación monetaria de dos millones de libras esterlinas

32 La ciudad de Cobija, capital del departamento de Pando, ocupa hoy en día el sitio de la antigua barraca de Bahía.

33 Fifer, 1966; 1970: 130.

34 El conflicto en el Acre puede ser dividido en, por lo menos en dos campañas (1899 y 1902) la segunda siendo apoyado por el gobierno del Estado de Amazonas con el resultado de la cesión del territorio Acreano al gobierno brasileño en 1903.

35 Fifer, 1966, Hecht - Cockburn, 1990: 79-86; Pacheco, 1992: 44.

36 Actualmente, el 11 de octubre es una de las fechas festivas más importantes del departamento de Pando, que ocupa la mayor parte del noroeste amazónico que, a inicios del siglo XX, estaba bajo jurisdicción del Territorio Nacional de Colonias en el Noroeste. Asimismo, varias calles y organizaciones radicadas en la actual Cobija, llevan por nombre referencias al conflicto y a sus protagonistas, como Bruno Racua, el flechero indígena cuyas flechas en llamas favorecieron la victoria, Nicolás Suárez y Federico Román, general que obtuvo un gran reconocimiento en el conflicto y, aún más, tras la polémica que mantuvo con el mencionado patrón gomero. En este sentido ver Suárez, 1928.

37 Suárez, 1928; Solares Arias, 1951.

38 En todo el territorio amazónico boliviano se perdieron un total de $190.000 \mathrm{~km}^{2}$ como resultado de este conflicto. Fifer 1970: 135 . 
para la república boliviana y la promesa de construir una vía ferroviaria destinada a superar los rápidos de agua -las llamadas cachuelas- más difíciles del río Madera. El principio de uti possidetis de facto, el derecho legal a la tierra para los que la ocupan y la usan, permitió al gobierno brasileño justificar su toma de posesión del Acre, apelando a la presencia de siringueros en la zona desde hacía largo tiempo ${ }^{39}$.

\section{Trabajadores de la Casa Suárez y el régimen de trabajo}

La atracción y retención de mano de obra fue una preocupación importante para la Casa Suárez desde su creación en la década de 1880. La rápida expansión de la economía gomera en un espacio únicamente ocupado por poblaciones no sometidas obligó al traslado de la mayoría de sus trabajadores desde otras regiones del país para contar con la mano de obra necesaria en la extracción del látex. Según el sociólogo Pablo Pacheco, el objetivo era crear "un mercado de trabajo regional" que mantuviera "equilibradas las necesidades de disponibilidad de mano de obra con la oferta interna conseguida con la incorporación de mano de obra extra-regional"40. El análisis de los documentos internos de la empresa Casa Suárez indica, sin embargo, que este equilibrio nunca llegó a establecerse. Si bien la industria gomera atrajo trabajadores procedentes de varias regiones de Bolivia y de muchas otras partes del mundo, los patrones gomeros raramente consiguieron que los grupos étnicos que vivían en los bosques de la zona se integraran a las barracas, e incluso cuando lo hicieron, nunca llegaron a cubrir la demanda cada vez más creciente de mano de obra en los numerosos asentamientos gomeros ${ }^{41}$. Esto ocasionó que durante la primera década del siglo XX prácticamente se agotaran los mercados de trabajo disponibles e, incluso, llegó a provocar problemas de despoblación en otras partes del Oriente boliviano ${ }^{42}$.

Una de las razones de la persistente escasez de mano de obra fue la elevada tasa de mortalidad de los trabajadores de las barracas gomeras. En los bajíos donde crece el árbol de siringa, las enfermedades transmitidas por mosquitos como la malaria, la fiebre amarilla y el beri beri son endémicas y fueron las responsables de la muerte de aquellos trabajadores poco acostumbrados al clima tropical. Las picaduras de serpiente, los ataques de animales silvestres, y los accidentes como la caída de árboles y el ahogamiento en los ríos también contribuyeron a un alto número de decesos ${ }^{43}$. Con el objetivo de escapar de las duras condiciones de trabajo y las altas deudas contraídas, algunos trabajadores huyeron a Brasil, donde la Casa Suárez no podía perseguirlos y, al mismo tiempo, las autoridades mostraban poca predisposición por devolver estos individuos al otro lado de la frontera ${ }^{44}$. El problema de la escasez laboral se agudizaría tras la crisis económica de la década de 1920, haciendo cada

39 Tocantins, 1961; Hecht - Cockburn, 1990: 79-86.

40 Pacheco, 1992: 70.

41 Ibídem, 61-67; Vallvé, 2010: 240.

42 Lema, 2009.

43 Existe mucha bibliografía que muestra los peligros y dificultades que enfrentaron a los trabajadores de siringa. Entre otros véase Coimbra, 2010 [1946]; Vallvé, 2010.

44 Denuncias de huidas se encuentran en la correspondencia de la Casa Suárez como cuando agentes de la empresa radicados en Villa Bella, en la frontera con Brasil, informaron de la huida de un grupo de trabajadores que se refugiaban en una barraca brasileña que no quería abandonar. Correspondencia reservada de la agencia de Villa Bella a la casa matriz. Cachuela Esperanza, 6-I-1926. ACS, Fondo Antiguo (en adelante FDA) No II, p. 5-338. 
vez más difícil el reclutamiento de trabajadores desde las fuentes habituales. En consecuencia, se puede afirmar que la atracción de una cantidad suficiente de trabajadores y la garantía de su permanencia en los lejanos centros de extracción gomera fueron problemáticas constantes que debieron afrontar sociedades comerciales como la Casa Suárez.

El típico trabajador de la Casa Suárez elude una sola definición o identidad coherente. De hecho, cabe recordar que la población en las áreas de producción gomera era sumamente internacional encontrándose, entre otros, alemanes, suizos, portugueses, brasileños, japoneses, sirios y libaneses que convivían con una población nacional también altamente diversa. La Casa Suárez originalmente dividió el trabajo por origen étnico y cualificación, pero con el tiempo la confianza, la lealtad y el buen desempeño en sus tareas se convirtieron en factores importantes de mejora laboral ${ }^{45}$. Suárez fue un gran admirador de la cultura suiza: individuos de esta nacionalidad fueron contratados como administradores de alto nivel, ingenieros, contadores y médicos en las barracas; trabajos parecidos fueron ocupados, también, por cruceños de clase alta con capacidades que eran apreciadas por el patrón de la sociedad comercial ${ }^{46}$. Sin embargo, estos trabajadores fueron una minoría si se compara con los numerosos indígenas y mestizos reclutados en los departamentos de Beni y Santa Cruz para trabajar en calidad de mano de obra no cualificada.

Dentro del régimen laboral de la Casa Suárez existía una gran pluralidad de arreglos laborales entre los trabajadores no especializados ${ }^{47}$. La mayoría de los trabajadores fueron empleados como mozos, una categoría laboral muy amplia que incluía varios tipos de trabajos y con la que la Casa Suárez identificaba los trabajadores endeudados con la empresa que carecían de contratos personales. Los mozos podían trabajar en los gomales, sujetos a las órdenes de los jefes de las barracas, tripular embarcaciones, o de agricultores. Por su parte, los siringueros -también conocidos como "picadores de goma"- se dedicaban exclusivamente a la pica de los árboles de las estradas gomeras que alquilaban a la empresa, su legal propietaria; práctica que, posteriormente, mantendrían los recolectores de castaña (también conocida como almendra del Brasil). Los fregueses estaban un eslabón por encima de los empleados mencionados porque aunque realizaban las mismas tareas que los siringueros, trabajaban por y para ellos mismos con un contrato independiente con la empresa, signo de confianza de la misma. Había también los llamados "alquilantes" que alquilaban estradas o barracas gomeras controladas por la Casa Suárez y actuaban como subcontratistas, con dominio de hasta una docena de estradas que eran trabajadas por unos pocos siringueros ${ }^{48}$. La mayor parte de estos trabajadores realizaban sus tareas de pica, recolección y deshumación del látex extraído de forma solitaria e independiente ${ }^{49}$. Todos ellos vivían con sus familias en los centros gomeros que se

45 Gamarra, 2007: 289-309.

46 Roca, 2001: 98-113; Córdoba, 2015.

47 Es importante señalar aquí que las categorías laborales fueron siempre muy fluidas, adaptándose al cambio de estaciones y a la meteorología, tal y como muestra Assies, 2002.

48 Pacheco, 1992: 85-92.

49 Este modelo de recolección funcionó en todas las áreas de producción de Hevea Brasilensis. Véase Santos, 1980; Weinstein, 1983; Pacheco, 1992; y Gamarra, 2007. Cabe recordar aquí que la extracción del caucho -que recibe el nombre científico de Castilla Ulei- en los bosques del norte del Perú, Colombia y parte del Brasil requería de otros métodos de extracción lo que, por ende, dio lugar a un sistema laboral totalmente distinto. 
hallaban dispersados al interior del bosque. Según el tamaño y la importancia de la barraca de la que dependían, éstos podían encontrarse en cualquier lugar entre unas dos horas de caminata y un viaje de un día completo desde dicho establecimiento. Este asentamiento disperso fue básico tanto para la ocupación territorial de la zona ante la ausencia de otro modelo o mecanismo impulsado por las autoridades competentes, como para un efectivo desenvolvimiento del régimen laboral que dominaba la región gomera.

Este régimen funcionaba según los principios de deuda y patronazgo. La mayoría de los trabajadores de las barracas gomeras fueron reclutados a través del sistema de "enganche". Los individuos que ejercían de enganchadores en los centros urbanos de las tierras bajas implementaron estrategias, en muchos casos deshonestas, para captivar trabajadores por medio del adelanto de sumas de dinero en efectivo o bienes. Una vez insertos en la economía de la goma elástica, su vínculo con la compañía quedaba mediado por la obligación a saldar dichos adelantos con su trabajo ${ }^{50}$. Si bien Stoian enfatiza la existencia de salarios, incluso para mozos y jornaleros de bajo nivel ${ }^{51}$, concuerdo con Assies ${ }^{52}$ cuando señala que no debe pensarse en un sistema basado en salarios, dado que las cuentas de la mayoría de los peones registraban deudas demasiado elevadas como para ser pagadas con esos sueldos $^{53}$. Así, en realidad el sistema funcionaba como una forma de peonaje por deuda, donde la mano de obra quedaba indefinidamente hipotecada con la empresa y obligada a saldar sus adeudos con el fruto de su trabajo ${ }^{54}$. Aunque la Ley de Enganches de 1896 estipuló que la deuda de un trabajador enganchado no podía sobrepasar los 400 bolivianos, en la práctica esta normativa casi nunca fue observada, de tal modo que, frecuentemente, los mozos y los fregueses tenían deudas con la Casa Suárez por montos superiores a los 1.000 bolivianos ${ }^{55}$. El patronazgo también formaba parte importante del régimen laboral de la Casa Suárez. La relación entre el trabajador y los administradores de la Casa se regía, muchas veces, por una especie de paternalismo en el que se mezclaban la autoridad y el respeto, con la protección y el cuidado de su gente. Un tipo de práctica que, como veremos a continuación, se activó de forma especial para hacer frente a la crisis gomera y a la inseguridad territorial que aún existe en la zona.

\footnotetext{
50 Roca, 2001: 98-113; Vallvé, 2012; Brass, 1990.

51 Stoian, 2000: 63.

52 Assies señala que en uno de los contratos citados por Stoian para mostrar que el sistema en la Casa Suárez se basaba en salarios, en realidad el grupo de indígenas al que hacía referencia recibía sólo una pequeña parte de salario, mientras que el resto era recibido por un tercero, un tipo de enganchador que contrataba los indígenas en las misiones y los traía para trabajar la goma. Assies, 2002: 95. El contrato mencionado se reproduce en Pacheco, 1992: 237.

53 Assies 2002: 98-99.

54 Pacheco, 1992: 80. Existían también casos de mozos y siringueros que ahorraban y pagaban sus deudas, hasta que llegaron a tener un saldo a su favor en su cuenta. En Córdoba, 2015: 107-112, se encuentra relatos dan cuenta de condiciones ventajosas para los trabajadores indígenas, pero hay que tener en cuenta que estos datos proceden de empleados europeos privilegiados que rememoraban sus vivencias en las barracas gomeras.

55 La voluntad de las autoridades bolivianas por asegurar la implementación de la Ley de Enganches para evitar que los trabajadores gomeros fueron víctimas de deudas ilegales fue la causa de ciertas fricciones entre el Estado boliviano y la Casa Suárez que vio como muchos de esos huían a los centros urbanos benianos para denunciar su situación irregular ante los poderes públicos, tal y como queda documentado en Cartas y circulares entre la casa matriz y varios establecimientos. Correspondencia reservada del establecimiento Porvenir a la casa matriz. Porvenir, 27-V-1927. ACS/FDA, No II, p. 5-338
} 


\section{Crisis y adaptación}

En 1920 la economía gomera en toda la Amazonía entró en una crisis de la que nunca se recuperaría completamente. Una década antes, las plantaciones gomeras del sudeste asiático habían inundado el mercado mundial provocando, así, el desplome del precio de este producto ${ }^{56}$. La industria boliviana siguió obteniendo ganancias a lo largo de esa década pero a partir de los años '20, la crisis se agudizó y la economía gomera colapsós ${ }^{57}$. Los valores de exportación de la goma boliviana cayeron de los 15 millones y medio de bs. en 1917 a los poco más de tres millones en 1921; y aunque hubo un repunte en 1926, los valores nunca superaron los de antes de $1920^{58}$. El mercado de la goma entró en una crisis más profunda tras el colapso económico mundial de 1929 y la guerra del Chaco (1932-1935) que provocó el retraimiento de la economía de la goma en la década de 1930. Esta situación forzó a la Casa Suárez a orientar una parte considerable de su negocio a la extracción de castaña, introduciendo las primeras máquinas de procesamiento de este producto, destinado a la exportación internacional ${ }^{59}$. La diversificación de sus actividades y un control estricto de capital acumulado durante los mejores años de la economía gomera permitieron a Nicolás Suárez mantener su imperio durante las difíciles décadas de 1920 y 1930, cuando la mayoría de los productores gomeros de la región ${ }^{60}$ se cesaron con sus actividades ${ }^{61}$.

Hasta la fecha, se ha estudiado la reestructuración de carácter económico y empresarial que realizaron las casas comerciales, particularmente la Casa Suárez, para hacer frente a la decadencia de la economía de la goma elástica en la Amazonía boliviana, entre 1920 y $1950^{62}$. Pero no existe todavía un estudio de carácter más etnográfico, tomando en cuenta los cambios en el sistema de trabajo que impuso la empresa, la motivación de tal mutación y la respuesta del personal administrativo a las nuevas condiciones sociales y laborales de los mozos, siringueros y fregueses. La incertidumbre que este nuevo contexto económico generó entre los administradores de la empresa y la preocupación que persistía en la región por los antecedentes bélicos que ahí se habían vivido -lo que Ann Laura Stoler denomina epistemic anxieties $^{63}$-, ocasionaron variaciones en la orientación, funcionamiento y prioridades de la Casa Suárez y una transformación de la percepción que, hasta la fecha, habían tenido de su mano de obra.

El resultado de la guerra del Acre -la pérdida de territorio nominalmente boliviano en el noreste y la evidencia de la debilidad estatal, tanto política como militar-, generó nuevas preocupaciones para la Casa Suárez relativas a la defensa de sus posesiones y de la bolivianidad en esa región fronteriza. Esa actitud fue, muy probablemente, influencia por las inquietudes expresadas por las autoridades gubernamentales. En las memorias presentadas por los delegados del Territorio Nacional de Colonias en el Noroeste al Ministerio de Colonización de 1921, 1922 y 1923 son

\footnotetext{
Tully, 2011.

Stoian, 2005: 75.

Gamarra, 2007: 108-109; Stoian, 2005: 75.

Fifer, 1970: 144; Pacheco, 1992: 96-99; Stoian, 2005: 86.

60 La Casa Seiler -antigua Casa Braillard- fue la única que, junto a los Suárez, supervivió a la crisis gomera y apostó por la industria castañera. Si bien Assies (2002: 124n) puntualiza que solo devino una potencia tras la desintegración de la Casa Suárez en la década de 1940.

${ }_{61}$ Fifer, 1970: 144.

62 Pacheco, 1992; Stoian, 2005; Gamarra, 2007.

63 Stoler, 2009.
} 
reiteradas las alusiones a la "más grave crisis que hasta ahora sufrieran el territorio de colonias y el Beni" ${ }^{64}$. Crisis en la que la mayor de las preocupaciones era el despoblamiento del noroeste boliviano ${ }^{65}$ tal y como se desprende de las palabras del delegado: "nuestra extensa frontera con el Brasil, la ausencia de población boliviana y la dolorosa experiencia que tenemos recogida, imponen no descuidar este asunto que es de vital importancia para el país, sin perder de vista que la soberanía solo es efectiva cuando la acción de los pobladores puede sostenerla" ${ }^{66}$. Su autor era consciente que la crisis económica en la región estaba provocando un éxodo de trabajadores y, por lo tanto, de pobladores, y urgía al gobierno a poner freno a este fenómeno para evitar que la frontera boliviana volviera a ser vulnerable a las agresiones brasileñas ${ }^{67}$. El problema del despoblamiento se encuentra, también, en las cartas intercambiadas entre el delegado del Territorio de Colonias del Noroeste y la Casa Suárez ${ }^{68}$. Refiriéndose a "la experiencia que tengo y que debemos tener todos los bolivianos, de los sucesos desgraciados conocidos en este territorio, con motivo de la revolución acreana" ${ }^{69}$ y apelando al patriotismo y al civismo de la empresa -y, especialmente, de su director Nicolás Suárez-, el delegado solicitaría que se emplearan en sus establecimientos más trabajadores bolivianos y se mantuvieran los existentes. En este sentido, el delegado llegó a sugerir a la Casa Suárez que en sus contratos de arrendamiento de estradas empezaran a imponer "la obligación ineludible de poner el 60\% de trabajadores nacionales [bolivianos] -entre empleados y obreros-, bajo pena de rescisión, pudiendo el $40 \%$ ser de otras nacionalidades" ${ }^{\prime 70}$.

Sin embargo, estas advertencias no eran necesarias. En el mismo año de 1920 la administración de la Casa Suárez envió una circular a varios de sus establecimientos en la que informaba a sus administradores que "[h] llegado el momento que nos obliga a tomar algunas disposiciones que se imponen por las circunstancias actuales, pues hoy día la goma se cotiza a lo que significa una enorme pérdida para nuestra Empresa"71. Estas disposiciones incluían un mayor impulso de la producción agrícola para el autoconsumo, la reducción de los gastos administrativos y de la compra de alimentos y bienes de lujo, y un esfuerzo para evitar el crecimiento de deudas demasiado elevadas en las cuentas de mozos y fregueses. Asimismo, el autor de la circular lamentaba que la mayor parte del personal de la Casa no era aún consciente de la gravedad de la situación económica del momento y señalaba que sólo lo entenderían al final del fabrico, cuando se percataran que el valor de la producción era mucho menor al esperado ${ }^{72}$. La necesidad de convencer a su personal de la nueva realidad da cuenta, no sólo del nerviosismo de los administradores de la empresa ante la cri-

\footnotetext{
64 Ministerio de Guerra y Colonización, 1921: 115.

65 Ello también quedó plasmado en la prensa del noroeste, valga de ejemplo, "Las guarniciones militares en el Noroeste. El despueble del Territorio de Colonia”. La Gaceta del Norte, nº 42, 22-XII-1922, 2-4.

66 Ministerio de Guerra y Colonización, 1921: 114.

${ }_{67}$ Como señala Fifer, para el Estado boliviano la pérdida del Acre no fue un problema suficientemente importante como para destinar más recursos a solucionar los problemas que enfrentaba la región del noroeste, demasiado alejada del centro de poder nacional en el altiplano. Fifer, 1972: 130.

68 Ver Correspondencia del establecimiento. Porvenir, años 1925-1926. ACS/FDA, No II, P 5-338.

69 Carta del delegado nacional en el Territorio de Colonias al establecimiento Porvenir. Cobija, 19-VI-1926. ACS/ FDA, No II, p. 5-338.

70 Ibídem.

71 Circular reservada de la casa matriz a los establecimientos. Cachuela Esperanza, 10-XI-1920. ACS/FDA, PQ/ SN No III A.

72 Ibídem.
} 
sis económica, sino que, al mismo tiempo, muestra como el año de 1920 marcó un drástico quiebre con el pasado.

Pensadas para sortear un problema puntual, estas medidas a corto plazo se convertirían en parte del nuevo modelo administrativo de la Casa Suárez en las dos siguientes décadas de decadencia de la economía gomera. Un modelo que fue reorientado hacia una posición más paternalista hacia su personal siringuero-fregues que, si bien aseguró la conservación de su fuerza laboral, repercutió negativamente en sus ganancias económicas ${ }^{73}$.

Entre 1920 y 1940, la mano de obra no cualificada de la Casa Suárez devino un recurso esencial para mantener pobladas sus posesiones en la frontera y, así, resguardarla contra posibles amenazas. En tanto que principal fuerza económica y social en la región, la Casa Suárez ya había demostrado en el conflicto del Acre su interés y necesidad por proteger la bolivianidad de la región. De ahí que, en los momentos de dificultad económica y en el que los fantasmas de la guerra reaparecían, la preservación de la fuerza laboral en sus extensos territorios deviniera la principal preocupación de la empresa. Por un lado, se quería proteger la inversión realizada, pues la mano de obra era la parte más costosa de sus operaciones ${ }^{74}$. Por otro lado, se la quería conservar en su lugar de trabajo al tratase de la única población que podía hallarse en muchas partes del noroeste boliviano ${ }^{75}$. En 1925, en una carta dirigida al delegado nacional en el Territorio de Colonias del Noroeste, la administración del establecimiento Porvenir escribía:

durante los últimos años de aguda crisis, [...] la Empresa de Suárez Hermanos ha pagado a sus trabajadores sumas que estaban muy por encima de los precios en Europa con el objeto de mantener sus personales, y evitar el despoblamiento de la región, y en la expectativa [sic] de que este sacrificio que ha costado ingentes sumas, seria recompensado con la continuación de los trabajos en mejores días, y con la satisfacción de haber tenido la reserva moral y material suficiente para dar vida a esta grande empresa y a la región entera ${ }^{76}$.

Es posible que, en su comunicación con el gobierno central, esta administración exagerara el sacrificio económico que la Casa Suárez estaba realizando para evitar el despoblamiento de la región y, con ello, pretendiera enfatizar la actitud patriótica de su dirección. Pese a ello, esta cita muestra claramente que la despoblación era un problema real para la empresa, que se vio obligada a tomar medidas para mantener a su gente -como ajustar precios de compra-venta de bienes y alimentos- que le causaron pérdidas económicas, situación que se mantuvo hasta la década de 1930. En una circular enviada por la casa matriz, en Cachuela Esperanza, a la administra-

73 Carta al delegado del Territorio Nacional de Colonias en el Noroeste. Porvenir, 28-VII-1925. ACS/FDA, No II, P 5-338.

74 Carta de Nicolás Suárez al ministerio de Guerra y Colonización. Ethea, 03-IX-1929. ACS/FDA, Libro de Cartas de Nicolás Suárez, SN.

75 Si bien usamos mayormente ejemplos del establecimiento Porvenir, esta problemática se puede comprobar en documentos referentes a otros establecimientos de la Casa Suárez. Ver Circular Reservada de la Casa Matriz en Cachuela Esperanza a varios establecimientos. Chachuela Esperanza, 10-XI-1920. ACS/FDA PQ/SN No III A.

76 Carta al delegado nacional en el Territorio Nacional de Colonias en el Noroeste. Porvenir. 28-VII-1925. ACS/ FDA, No II, p. 5-338. 
ción del establecimiento Porvenir se afirmaba que: "[e]1 problema para nos[otros] radica principalmente en mantener el dominio y vigilancia en nuestras pertenencias gomeras y conservar en ellas a nuestros trabajadores a un costo tal que nos permita subsistir la crisis actual"77. El control sobre todas las posesiones gomeras de la empresa era difícil de conseguir en cualquier situación, pero se hizo aún más desafiante durante los años de la crisis. Los trabajadores dedicados a la pica de la goma vivían en las áreas de recolecta del látex, alejados de los grandes centros de población y de las vías de transporte, y mantenían su casa y su familia mediante la entrega de bolachas de goma elástica al patrón a cambio de víveres y artículos de primera necesidad. La dramática caída del precio de la goma estimuló, a partir de 1920, el abandono de muchos centros y establecimientos gomeros por parte de sus trabajadores. Como anota Gamarra ${ }^{78}$ no existen datos suficientes para saber exactamente cuántas barracas fueron abandonadas ni cuántos trabajadores se retiraron de sus puestos. Sin embargo, la frecuencia con la que este hecho es mencionado en los documentos de la Casa Suárez y en las memorias presentadas por el delegado del Territorio Nacional de Colonias en el Noroeste da cuenta de la enorme inquietud que esto generaba entre las máximas autoridades (estatales y empresariales) de la zona ${ }^{79}$. Sin la fuerza laboral de la frontera no hubiera habido modo de "mantener el dominio y vigilancia" de la misma. De esta manera sólo existió una única manera de ejercer algún tipo de autoridad sobre el territorio: asegurarse que la gente siguiera viviendo ahí.

La Casa Suárez empleó varias estrategias para conservar su personal en sus lugares de trabajo que implicaron un cambio de percepción de las autoridades de la empresa respecto su mano de obra. Si a lo largo del boom gomero (1850-1920), la producción y la supervivencia eran responsabilidad exclusiva de los propios trabajadores, a partir de entonces éstos empezaron a ser considerados elementos a "conservar”. Surgió así una lógica paternalista un poco más asistencialista.

En 1931, la administración de la Casa Suárez decidió suspender completamente la explotación de la goma debido al bajo precio al que se vendía el producto en el mercado internacional. Sin embargo, antes de hacer oficial esta medida, la casa matriz de Cachuela Esperanza avisó al personal administrativo del establecimiento Porvenir que "[e]l precio [de la venta de la goma] no da a los fregueses para alimentar sus familias. Tenemos [la] obligación de suplementar la actividad de sus chacos antes de [la] suspensión completa de la pica para que tengan razón de permanecer dónde sus chacos, y no queden sin medios" $"$. Aquí, el uso del término "obligación" es notable en tanto que muestra la transformación de la figura del trabajador en el imaginario de los administradores de la Casa Suárez. Si anteriormente durante el auge gomero las relaciones de trabajo entre trabajador (mozo, freguez, siringuero, obrero) y la administración se caracterizaban como de compadrazgo asimétrico, como señala Córdoba $^{81}$, tras la crisis económica de 1920 la actitud paternalista se extendió más

77 Circular reservada de la Casa Matriz en Cachuela Esperanza al Establecimiento Porvenir. Cachuela Esperanza, 17-II-1931. ACS/FDA, Caja 82, sp.

78 Gamarra, 2007: 205.

79 Correspondencia reservada del Establecimiento Porvenir a la Casa Matriz en Cachuela Esperanza. Porvenir, 19-I-1927. ACS/FDA, No II, p. 5-338. Circular Reservada de la Casa Matriz en Cachuela Esperanza al Establecimiento Porvenir, Cachuela Esperanza, 17-II-1931. ACS/FDA, Caja 82, sp. Ver también Ministerio de Guerra y Colonización de Bolivia, 1921: 114; 1922: 34; 1928: 112.

${ }_{80}$ Circular reservada de la casa matriz al establecimiento Porvenir. Cachuela Esperanza, 17-II-1931. ACS/FDA, Caja 82, SP.

81 Córdoba, 2012. 
profundamente al convertirse en una fuerte razón para asegurar la permanencia de la fuerza laboral, llegando, incluso, a reemplazar las razones puramente económicas ${ }^{82}$. A partir de entonces ya no se hablará de la obligación de producir suficiente cantidad de goma que tenían los mozos, siringueros y fregueses con la Casa, sino de la obligación de esta última con su fuerza laboral. Esta inversión en la percepción respondía, obviamente, a la escasez de mano de obra en la región tras la crisis gomera y que se agudizaría al iniciarse la guerra del Chaco: muchos se negaban a engancharse para trabajar en los gomales; otros se encontraban en territorio brasileño y no pensaban regresar; y muchos jóvenes se enrolaron en las tropas que defendieron la patria en el sureste del país ${ }^{83}$. Esta situación favoreció que, de algún modo, la supervivencia de la Casa Suárez, que desde el boom de la economía gomera habían ostentado el control absoluto sobre su mano de obra, quedara a merced de esta última que se revalorizó por su experiencia, tanto en el trabajo como por su adaptación al tipo de vida que requería la región; asimismo, la mejora de condiciones como la liquidación de las deudas más elevadas favoreció que los trabajadores optaran por quedarse y la empresa tuviera una mayor posibilidad de recuperar su inversión ${ }^{84}$.

En un contexto de una crisis económica generalizada por toda la región, la Casa Suárez reorientó sus prioridades económicas con el objeto de ofrecer una mayor estabilidad a sus trabajadores que fomentara su permanencia en la zona e, incluso, atrajera a nuevo personal. Era, entonces, necesario garantizar su acceso a la agricultura y a la producción de alimentos ${ }^{85}$. Las escasas rentas obtenidas con la comercialización de la goma dificultaban la importación de comida desde otras partes del país, favorecieron la reorientación de las fuerzas productivas ${ }^{86}$. Antes de la crisis, algunos establecimientos de la Casa Suárez ocupaban parte de sus trabajadores al cultivo de algunos chacos para producir alimentos para el autoconsumo. Sin embargo, estas actividades económicas eras minoritarias. Durante los años de auge, la Casa generalmente había disuadido, cuando no prohibido, a su personal trabajar sus propios cultivos dado que la lógica empresarial imponía la producción de goma como máxima prioridad ${ }^{87}$. Las nuevas directrices de la empresa incentivaron la producción de sus propios chacos entre los mozos, siringueros y fregueses. Este cambio de lógica

82 Agradezco los comentarios de uno de los evaluadores anónimos relativos al compadrazgo en la empresa gomera el cual, evidentemente, no fue un fenómeno nuevo de la década de 1920, pero sí devino un aspecto fundamental en las relaciones laborales de la región tras la crisis económica.

83 La Casa Suárez desde su inicio tenía el problema de escasez laboral, pero en los años posteriores a 1920 este problema se profundizaba gravemente. Véase Córdoba, 2012; Vallvé, 2010; Stoian, 2000; Gamarra, 2007; Pacheco, 1992; Roca 2001.

84 Cabe señalar que los problemas con los nuevos trabajadores eran comunes. Por ejemplo, en Porvenir, a mediados de la década de 1920 muchos de los fregueses y mozos que llegaron no se adaptaron bien a las condiciones de su nuevo trabajo: aquellos que no cayeron enfermos durante el viaje, se enfermaron en la barraca; otros se negaron a hacer un trabajo tan sacrificado como la recolección de la goma; otros buscaron la protección de las autoridades de Cobija, por entonces capital del Territorio Nacional de Colonias del Noroeste, donde acusaron a la Casa de no cumplir con sus contratos; otros huyeron a Brasil. Estos acontecimientos son un ejemplo de algo que pasaba con regularidad y causaba bastante angustia para funcionarios de la Casa Suárez. Cartas reservadas a Cachuela Esperanza. Porvenir, 17-XII-1926 - 19-III-1927. ACS/FDA, No II, p. 5-338.

85 Circular reservada de la casa matriz a los establecimientos. Cachuela Esperanza, 10-XI-1920. ACS/FDA, PQ/ SN No III A.

86 Ibídem.

87 La producción de alimentos en la Casa Suárez varió bastante según el momento histórico y el lugar en el que se encontraba cada establecimiento, pero podemos afirmar que durante los años de mayor explotación de la goma elástica -y, por consiguiente, de mayor crecimiento económico de la empresa-, la Casa apostó por desincentivar cualquier actividad económica no relacionada directamente con la producción gomera. 
se debió a dos motivos. Uno respondía al interés por fomentar su autonomía y hacerles más autosuficientes para, así, evitar emplear los reducidos recursos de la Casa en este asunto ${ }^{88}$. Ello suponía una transformación parcial de la administración del mundo laboral y económico que había primado en la empresa hasta la fecha y en la que se fomentaba la dependencia absoluta del trabajador: no sólo debían vender la cantidad de goma que recolectaban a un único patrón, también debían abastecerse de artículos de primera necesidad -particularmente alimentos- en la única tienda que existía en cada barraca y a precios desorbitados. El otro motivo pretendía arraigar a los trabajadores en su lugar de trabajo, ya no tanto por medio del endeudamiento del trabajador -tal y como se había caracterizado el período anterior y por el cual se lo mantenía "enganchado" a la empresa y al lugar de trabajo-, sino pensando que la labor y el esfuerzo invertido en el cultivo de sus chacos les vincularía aún más con la empresa e impediría el abandono de la misma ${ }^{89}$. Con la disminución de la recolección de goma elástica, principal actividad económica del norte amazónico boliviano, el sistema de endeudamiento dejó de tener sentido y la administración de la Casa Suárez tuvo que buscar nuevas fórmulas para enraizar a los trabajadores en las lejanas barracas gomeras.

Como señalara Gamarra ${ }^{90}$ la crisis económica que se inició en 1920 impidió que la Casa Suárez ejerciera el férreo control sobre su personal que, a pesar de las grandes distancias que mediaban al interior de este imperio empresarial y la naturaleza solitaria del trabajo siringuero, había desplegado anteriormente ${ }^{91}$. Por un lado, los limitados recursos administrativos con los que, a partir de ahora, contaba la empresa dificultaban logísticamente todo control laboral estricto. Por otro lado, la nueva lógica buscaba que los trabajadores quisieran quedarse a vivir y trabajar en las posesiones territoriales de la Casa y para conseguirlo la administración se afanó por extender unas condiciones suficientemente favorables para toda su población de trabajadores $^{92}$. Cuando en 1931 se suspendió la explotación de la goma, la casa matriz instruyó a sus establecimientos a "dejar en libertad los trabajadores para que se dedican [sic] a lo que quieran dentro de nuestras propiedades, aunque vendan sus productos solo a la Casa y no a terceros"93. Su objetivo era claro: hacer de "nuestra gente" trabajadores asociados con la Casa Suárez para que se quedaban en sus lugares de trabajo y, por consiguiente, los territorios de la empresa no fueran abandonados. Este no era un problema nuevo. Desde antes de la crisis económica iniciada en 1920, la Casa Suárez ya había mostrado su preocupación por asegurar la permanencia de la mano de obra en sus lugares de trabajo, dado que si un mozo o un freguez huía -pero también si enfermaba o se accidentaba- la Casa tendría dificultades para cobrar lo

88 Emergencia anteproyecto. Circular reservada de la casa matriz a varios establecimientos. Cachuela Esperanza, 28-III-1931. ACS/FDA, Caja 82, sp.

89 Circular reservada de la casa matriz al establecimiento Porvenir. Cachuela Esperanza, 17-II-1931. ACS/FDA, Caja 82, sp.

90 Gamarra, 2007: 210.

91 Las principales investigaciones que han abordado la economía gomera y su estricto sistema laboral son Pacheco, 1992; Roca, 2001: 98-113; Gamarra 2007, Vallvé 2010, y Stoian 2000. La literatura también ha recogido experiencias de este sistema como en Coimbra, 2010 [1946]; Durán Böger, 1967.

92 Circular reservada de la casa matriz a los establecimientos. Cachuela Esperanza, 10-XI-1920. ACS/FDA, PQ/ SN No III A.

93 Emergencia anteproyecto. Circular reservada de la casa matriz a varios establecimientos. Cachuela Esperanza, 28-III-1931. ACS/FDA, Caja 82, sp. 
que se le adeudaba ${ }^{94}$. Por este motivo, la empresa desplegó todos los recursos que tenía a mano para evitar tales pérdidas. Sin, embargo, tras la crisis y, especialmente, tras la suspensión de la explotación de la goma elástica a partir de 1931, los administradores de la Casa se vieron obligados a cambiar de estrategia. Ahora era necesario convencer a su gente para que se quedaran en sus lugares de trabajo, una tarea mucho más difícil de la que habían enfrentado hasta la fecha.

\section{Conclusiones}

La bibliografía sobre el auge de la goma elástica en la Amazonía es abundante, pero mucho menos se ha escrito sobre el periodo posterior, entre 1920 y 1940, en el que se vivió la decadencia de la economía y sociedad gomeras ${ }^{95}$. Este vacío historiográfico responde a la consiguiente disminución de las actividades vinculadas a la extracción y comercialización del látex y, por ende, de la despoblación de siringueros en el espacio amazónico continental, lo que dificulta el acceso a fuentes seriadas para este período. En países como Brasil, Perú y Colombia, los productores de goma elástica acabaron abandonando sus actividades después de $1920^{96}$. Esto, sin embargo, no ocurrió con la misma intensidad en Bolivia donde, como señalan varios autores ${ }^{97}$ algunas de las grandes empresas gomeras, y en particular la Casa Suárez, sobrevivieron a los ciclos de decadencia en la economía gomera, convirtiendo la experiencia boliviana en una excepción digna de ser estudiada con mayor detalle. El caso de la empresa de Nicolás Suárez muestra, no sólo las formas de adaptación para sortear crisis económicas y políticas, sino también el sistema laboral y patronal impuesto y reformulado con el paso el tiempo, cuyo estudio es esencial para comprender la realidad contemporánea de la vida social y económica de la región ${ }^{98}$.

Retomando la propuesta analítica de Ann Laura Stoler, en este trabajo he estudiado el fondo histórico de la Casa Suárez como un registro de una economía emocional -entendida como incertidumbres y dudas- que manifestaba diferentes interpretaciones de lo que se había vivido y presenciado, se imaginaba y se temía; desaliento, recelos y expectativas sobre el modo en que el mayor imperio gomero boliviano debía reaccionar ante un contexto de cambio que fueron plasmados en la documentación de la empresa ${ }^{99}$. En vez de analizar los datos numéricos y los acontecimientos literalmente descritos en la documentación, la metodología etnográfica planteada por esta autora propone el análisis de las actitudes y las emociones que se esconden detrás de la producción de las fuentes. En este sentido, el uso de esta metodología me ha permitido examinar las mentalidades de la gerencia y dirección de la Casa Suárez entre 1920 y 1940. Los distintos documentos de la empresa ofrecen ejemplos notables de declaraciones, descripciones, órdenes y quejas que revelan cómo la memoria del pasado bélico - la guerra del Acre-y la presente convulsión económica -crisis de

Coimbra, 2010 [1946]; Córdoba, 2015.

95 Weinstein en su texto clásico sobre el auge de la goma, afirma que después de 1920 la región amazónica quedó estancada, con excepción de un pequeño auge durante la Segunda Guerra Mundial. Weinstein, 1983.

96 Bradford - Coomes, 1996; Weinstein, 1983; Santos, 1980; Stanfield, 1998.

97 Assies, 2002; Fifer, 1970; Pacheco, 1992; Stoian, 2005; Gamarra, 2007.

98 Pacheco, 1992; Assies, 2002.

99 Stoler, 2009: 4. 
la producción de goma elástica en la Amazonía- condicionaron la manera en que la Casa concebiría a sus trabajadores.

Durante los años de crisis en la economía gomera, la Casa Suárez recurrió a varias estrategias nuevas para poder sobrevivir. Uno de los problemas más urgentes que tuvo que afrontar fue el mantener a la fuerza laboral no cualificada dispersada por sus extensas pertenencias gomeras. La falta de mano de obra y la amenaza a un abandono sistemático de la región por parte de los trabajadores existentes debido a los bajos precios de goma elástica obligaron a una reorientación en sus prioridades y al desarrollo de una nueva percepción sobre este colectivo. Esta nueva lógica de la Casa se evidenció con las directrices que impedían a la administración de los establecimientos asegurar la alimentación y condiciones de vida de sus mozos, siringueros y fregueses -así como de sus familias-, dejando en un segundo plano cualquier preocupación sobre las deudas que corrían por cuenta de los trabajadores. En efecto, a partir de 1920, el personal no especializado empezó a ser concebido como un grupo que debía ser cuidado y protegido. Si bien este cambio pretendía frenar el impacto altamente negativo que supondría la pérdida de su mano de obra, sostenemos aquí que la voluntad por procurar el bienestar de los trabajadores resultó de la notable inquietud que generaba en la dirección de la Casa la preservación de la integridad territorial, tanto de la empresa, como de la propia república boliviana.

El "dominio y vigilancia" de las pertenencias de la Casa Suárez dependían básicamente de su habilidad en mantener los territorios poblados, en especial para el establecimiento de Porvenir y sus dependencias, las más cercanas al límite fronterizo con Brasil. La dramática experiencia vivida en la guerra del Acre por Nicolás Suárez tuvo serias repercusiones en la administración posterior de la empresa, donde las inquietudes y preocupaciones relativas a la inseguridad en la zona de frontera y la incertidumbre ante nuevas posibles agresiones territoriales por naciones vecinas se convirtieron en política empresarial, tal y como se desprende de sus documentos. En este sentido, la lectura de estas fuentes desde la perspectiva de la etnografía de archivo permite observar los efectos que pervivían en la psique colectiva relativos a las pérdidas territoriales y los enfrentamientos militares para comprender de forma holística las razones, tanto emocionales como más racionales, que existían detrás de las políticas empresariales y sus implicaciones.

\section{Referencias bibliográficas}

Assies, Willem. "From Rubber Estate to Simple Commodity Production: Agrarian Struggles in the Northern Bolivian Amazon". The Journal of Peasant Studies, vol. 29, n 3-4 (2002): 83-130. DOI: 10.1080/03066150412331311039

Brass, Tom. "The Latin American Enganche System: Some Revisionist Reinterpretations Revisited". Slavery \& Abolition, vol. 11, no 1 (1990): 74-103. DOI: 10.1080/01440399008575001

Chartier, Roger. Cultural History: Between Practices and Representations. Ithaca: Cornell University Press, 1988.

Coimbra, Juan B. Siringa: memorias de un colonizador del Beni [1946]. La Paz: Librería Editorial "GUM," 2010.

Córdoba, Lorena I. (ed.). Dos suizos en la selva: historias del auge cauchero en el Oriente boliviano. Santa Cruz de la Sierra, Solidar-Suiza-CIHA, 2015. 
- "Misiones-patrones e indígenas-siringueros: el caucho entre los chacobos del Beni (siglo XX)". Boletín Americanista, $\mathrm{n}^{\circ} 65$ (2012): 85-106.

Costa Sobrinho, Pedro Vicente. Capital e trabalho na Amazônia Ocidental: contribuição à história social e das sindicais no Acre. São Paulo-Rio Branco: Universidade Federal do Acre, 1992.

Cunha, Euclides da. Um paraíso perdido: reunião dos ensaios amazônicos. Petrópolis: Editora Vozes, 1976.

Durán Böger, Luciano. En las tierras de Enín. La Paz: Ed. Novedades, 1967.

Fifer, J. Valerie. "Bolivia's Boundary with Brazil: A Century of Evolution”. The Geographical Journal, vol. 132, no 3 (1966): 360-372. DOI: 10.2307/1793878.

- "The Empire Builders: A History of the Bolivian Rubber Boom and the Rise of the House of Suárez". Journal of Latin American Studies, vol. 2, nº 2 (1970): 113-46.

- Bolivia: Land, Location, and Politics since 1825. Cambridge: Cambridge University Press, 1972.

Gamarra, María del Pilar. Amazonía norte de Bolivia: economía gomera (1870-1940): bases económicas de un poder regional, la casa Suárez. La Paz: Colegio Nacional de Historiadores de Bolivia - Producciones CIMA Editores, 2007.

García Jordán, Pilar. Cruz y arado, fusiles y discursos: la construcción de los Orientes en el Perú y Bolivia, 1820-1940. Lima: Instituto Francés de Estudios Andinos - Instituto de Estudios Peruanos, 2001.

Guiteras Mombiola, Anna. De los llanos de Mojos a las cachuelas del Beni 1842-1938: conflictos locales, recursos naturales y participación indígena en la Amazonía boliviana. Cochabamba: Instituto de Misionología - Ed. Itinerarios - Archivo y Biblioteca Nacionales de Bolivia, 2012.

Hecht, Susana B. - Alexander Cockburn. The Fate of the Forest. Developers, Destroyers, and Defenders of the Amazon. New York: Harper and Row, 1990.

Lema, Ana María. El sentido del silencio. La mano de obra chiquitana en el Oriente boliviano a principios del siglo XX. Santa Cruz de la Sierra: El País - UPIEB, 2009.

Martinello, Pedro. A "batalha da borracha" na Segunda Guerra Mundial. Rio Branco: EDUFAC, 2004.

Ministerio de Guerra y Colonización de Bolivia, Delegación Nacional en el Territorio de Colonias. Informe que Informe que eleva al Ministerio de Guerra y Colonización el Delegado Nacional en el Territorio de Colonias. La Paz: s.p.i., 1921.

- Informe que eleva al Ministerio de Guerra y Colonización el Delegado Nacional en el Territorio de Colonias. La Paz: s.p.i., 1922.

- Informe que eleva al Ministerio de Guerra y Colonización el Delegado Nacional en el Territorio de Colonias. La Paz: s.p.i., 1923.

- Informe que eleva al Ministerio de Guerra y Colonización el Delegado Nacional en el Territorio de Colonias. La Paz: s.p.i., 1928.

Pacheco, Pablo. Integración económica y fragmentación social: el itinerario de las barracas en la Amazonia boliviana. La Paz: CEDLA, 1992.

Roca, José Luis. Economía y sociedad en el Oriente boliviano (siglos XVI-XX). Santa Cruz: COTAS, 2001.

Santos, Roberto. História Econômica Da Amazônia, 1800-1920. São Paulo: T.A. Queiroz, 1980.

Stanfield, Michael Edward. Red Rubber, Bleeding Trees: Violence, Slavery, and Empire in Northwest Amazonia, 1850-1933. Albuquerque: University of New Mexico Press, 1998. 
Scott, James. Weapons of the Weak. Everyday Forms of Peasant Resistance. New Haven: Yale University Press, 1985

Solares Arias, Napoleón. Síntesis biográfica del señor don Nicolás Suárez. Cachuela Esperanza: s.p.i., 1951.

Stoian, Dietmar. "Variations and Dynamics of Extractive Economies: The Rural-Urban Nexus of Non-Timber Forest Use in the Bolivian Amazon". Tesis Doctoral, Universitätsbibliothek Freiburg, 2000.

- La economía extractivista de la Amazonia norte Boliviana. Bogor Bara: Center for International Forestry Research, 2005.

Stoler, Ann Laura. Along the Archival Grain: Epistemic Anxieties and Colonial Common Sense. Princeton: Princeton University Press, 2009.

Suárez, Nicolás. Anotaciones y documentos sobre la campaña del Alto Acre, 1902-1903. Barcelona: Tip. La Académica, 1928.

Tocantins, Leandro. Formação Histórica Do Acre, vol. 5, Temas Brasileiros. Rio de Janeiro: Conquista, 1961.

- Euclides da Cunha e o paraíso perdido: tentativa de interpretação de uma presença singular na Amazônia e a conseqüente evolução de um pensamento sobre a paisagem étnico-cultural, histórica e social brasileira, alargando-se nos horizontes da história transcontinental, 3. ed. Coleção Retratos Do Brasil vol. 105. Rio de Janeiro: Civilização Brasileira, 1978.

Trouillot, Michel-Rolph. Silencing the Past: Power and the Production of History. Boston: Beacon Press, 1997.

Tully, John. The Devil's Milk: A Social History of Rubber. New York: NYU Press, 2011.

Vallvé, Frederic. "The Impact of the Rubber Boom on the Bolivian Lowlands (1850-1920)". Tesis Doctoral, Georgetown University, 2010.

- "La barraca gomera boliviana: etnicidad, mano de obra y aculturación (1880-1920)". Boletín Americanista, $\mathrm{n}^{\circ} 65$ (2012): 61-84.

Van Valen, Gary. Indigenous Agency in the Amazon. The Mojos in liberal and rubber-boom Bolivia, 1842-1932. Tucson: The University of Arizona Press, 2013. 